\title{
MAGNETORESISTANCE AND MAGNETIC PROPERTIES OF ANNEALED NiFeN THIN FILMS
}

\author{
B. ELMRABAT and Th. J.A. POPMA \\ University of Twente, Departments of Electrical Engineering and Applied Physics, P.O. Box 217, 7500 AE Enschede, \\ The Netherlands
}

Received 20 November 1989

\begin{abstract}
As-sputtered and annealed NiFe thin films were investigated by transmission electron microscopy (TEM), and Auger electron spectroscopy (AES). Their magnetoresistive and magnetic properties were characterized. It was found that both the microstructure and the physical properties of NiFe are affected by nitrogen addition in as-sputtered and annealed films. The out-diffusion of nitrogen during annealing strongly affects these properties and both the magnetoresistance and magnetic properties depend on the initial nitrogen content of the film.
\end{abstract}

\section{Introduction}

Metalloid elements are known as effective species in nucleation and growth processes of thin films. In NiFe, Jhingan [1] found that the microstructure is significantly influenced by the addition of nitrogen. An increase in the nitrogen content of the sputtering gas enhances the amount of $(002)$ crystal orientation and decreases the grain size. Krush [2] used an $\mathrm{Ar}-\mathrm{N}_{2}$ sputtering gas mixture and studied the magnetic properties of permalloy films. The most relevant result of this investigation is the effect of nitrogen on the saturation magnetostriction.

The above publications gave clear evidence of the effect of nitrogen on thick permalloy (1-1.5 $\mu \mathrm{m})$ film structures and magnetic properties. For some practical applications like transducers based on the magnetoresistive effect, the tendency is to use thinner $25-50 \mathrm{~nm}$ annealed films in order to increase the signal-to-noise ratio and to recover the electrical properties [3]. In thinner films the coercive force and the resistivity are much higher. The thickness dependence of the coercive force was recently attributed to inhomogeneities of chemical composition and impurities $[4,5]$. However, no systematic study has been performed to clarify the role of a specific metalloid element in very thin films.

Moreover the study of ternary systems based on $\mathrm{Ni}_{82} \mathrm{Fe}_{18}$ is of extreme importance for understanding the anisotropic magnetoresistance effect. While the impurity contribution of $\mathbf{3 d}$ transition metals has been investigated [6], there have been no studies on the contribution of the metalloid elements.

In this paper, we report the results of our investigation on the effect of the presence of nitrogen on the magnetic and magnetoresistive properties in as-deposited and annealed thin permalloy films.

\section{Experimental procedure}

Thin permalloy films (25 and $50 \mathrm{~nm}$ ) used in this investigation were deposited by rf sputtering apparatus from a $\mathrm{Ni}_{82} \mathrm{Fe}_{18}$ target in $\mathrm{Ar}, \mathrm{Ar} 1 \% \mathrm{~N}_{2}$, Ar $3 \% \mathrm{~N}_{2}$, Ar $5 \% \mathrm{~N}_{2}$ plasmas. After reaching a background pressure of $1 \times 10^{-5} \mathrm{~Pa}$, sputtering was performed in a total gas pressure of $25 \times 10^{-1}$ $\mathrm{Pa}$ keeping the target voltage at $800 \mathrm{~V}$ and the substrate bias at $80 \mathrm{~V}$. Depositions were made on thermally grown $\mathrm{SiO}_{2}, \mathrm{Si}$, and LPCVD $\mathrm{SiN}$ at a 
rate of $0.17 \mathrm{~nm} / \mathrm{s}$. The film thickness was controlled by using high-resolution SEM and AES depth-profile analysis methods. Annealing steps at $400^{\circ} \mathrm{C}$ for $900 \mathrm{~s}$ were performed in high vacuum $\left(10^{-5} \mathrm{~Pa}\right)$ to prevent oxidation and to ensure measurement reproducibility. Heating and cooling rates were chosen carefully to reduce the substrate stresses. The anisotropic magnetoresistive parameter $\Delta \rho$, the anisotropy field strength $H_{\mathrm{k}}$, and the easy-axis orientation were measured using the magnetoresistive method described in ref. [7]. The saturation magnetization and easy-axis coercive force were measured with a magnetic hysteresis loop tracer. In the case of hard magnetic materials we performed the measurements with a vibrating sample magnetometer VSM. The NiFe thin-film microstructure was investigated by transmission electron microscopy using a Jeol $200 \mathrm{CX}(200 \mathrm{kV})$. TEM specimens were prepared adopting a simple, and cleaner method [8]. Its principle consists of the deposition of the thin film to be investigated on a set of thin membranes. Its main advantage is the fact that the contaminating steps, like etching or substrate dissolving, are not necessary after sputtering. The chemical composition analysis of different films was performed using the AES method with a PHI 600 Scanning Auger Microprobe. Depth profiles were recorded using Ar sputter eroding and simultaneously performing AES analysis. Typical Auger traces covering a range of $30-2000 \mathrm{eV}$ were taken on the surfaces of as-deposited and annealed films.

\section{Results}

\subsection{Magnetoresistive measurements}

The effect of nitrogen contamination on $\Delta \rho$, the anisotropic magnetoresistance parameter, is given in fig 1 . In as-sputtered films $\Delta \rho$ degrades drastically with increasing nitrogen gas content: Films sputtered in an Ar $5 \% \mathrm{~N}_{2}$ gas mixture show no anisotropic magnetoresistance effect, as they are considered to be non-ferromagnetic. During annealing nitrogen diffuses out (see section 3.3) leading to the observed recovery of the considered electrical parameter $\Delta \rho$, which surprisingly de-

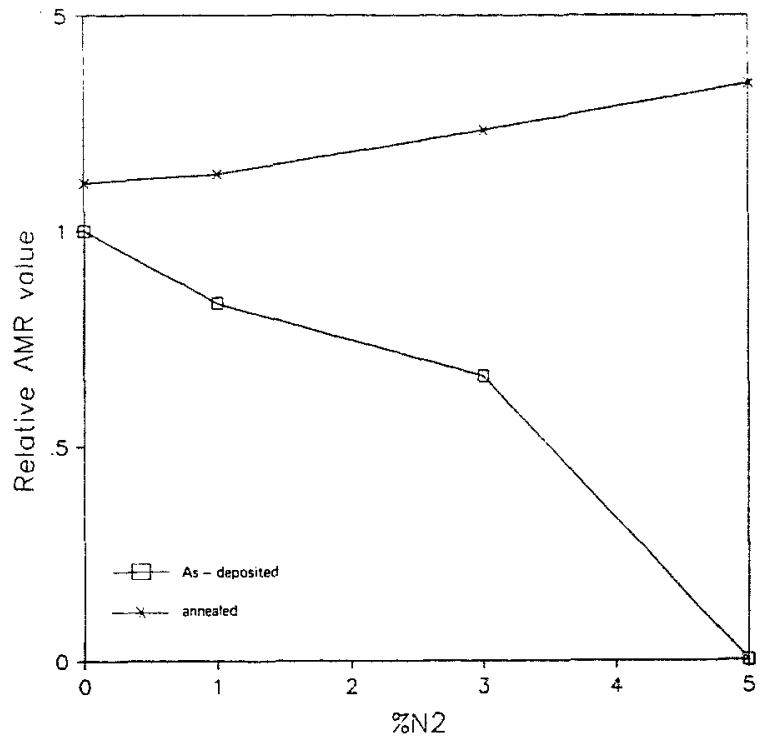

Fig. 1. The dependence of the relative anisotropic magnetoresistive parameter $\Delta \rho / \Delta \rho(\mathrm{NiFe})$ on the nitrogen sputtering gas content, other deposition parameters being constant.

pends on the initial nitrogen gas content. It increases linearly with the percentage of nitrogen. Annealed NiFe films sputtered in an Ar $5 \% \mathrm{~N}_{2}$ gas mixture exhibit a $\Delta \rho$ value $35 \%$ higher than in films sputtered in pure argon.

\subsection{Magnetic measurements}

The changes in the anisotropy field strength with thickness and nitrogen contamination are presented in fig. 2. The results of Krush [2] are also reported. It can be seen from this figure that $H_{\mathrm{k}}$ decreases with decreasing film thickness or increasing nitrogen content. The effect of nitrogen on $H_{\mathrm{k}}$ degradation is more pronounced in thinner films; a drop of $\boldsymbol{H}_{\mathrm{k}}$ from 300 to $70 \mathrm{~A} / \mathrm{m}$ was observed in films of $25 \mathrm{~nm}$ thickness sputtered in Ar $3 \% \mathrm{~N}_{2}$. Films sputtered in an argon $5 \% \mathrm{~N}_{2}$ gas mixture were magnetically isotropic. The annealing step reduces the dispersion of this parameter without affecting its mean value.

The other magnetic properties were also affected by the presence of nitrogen. Both the coercivity and the saturation magnetization decrease in as-deposited films when the nitrogen content 


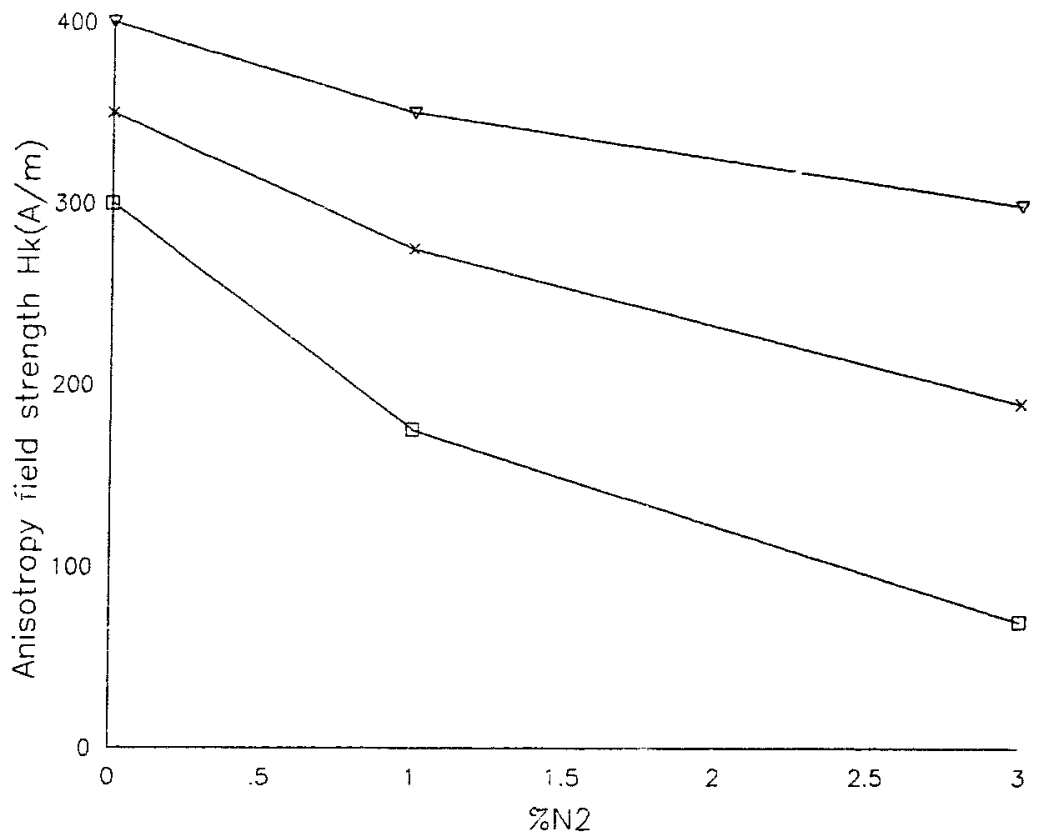

Fig. 2. The dependence of the anisotropy field strength $H_{\mathrm{k}}$ on the nitrogen sputtering gas content and NiFe film thickness. $\square: 25 \mathrm{~nm}, x: 50 \mathrm{~nm}, \nabla: 1.5 \mu \mathrm{m}$ (see ref. [2]).

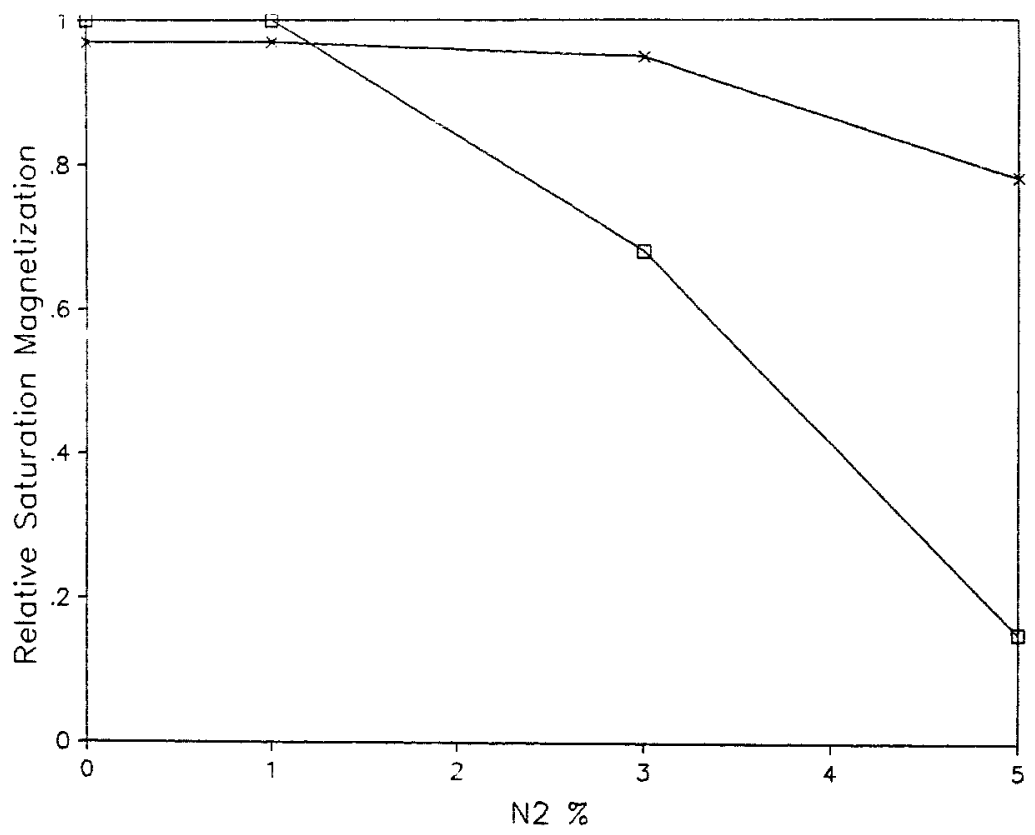

Fig. 3. The dependence of the relative saturation magnetization on the nitrogen sputtering gas content. $\square:$ as-sputtered, $\times$ : annealed. 


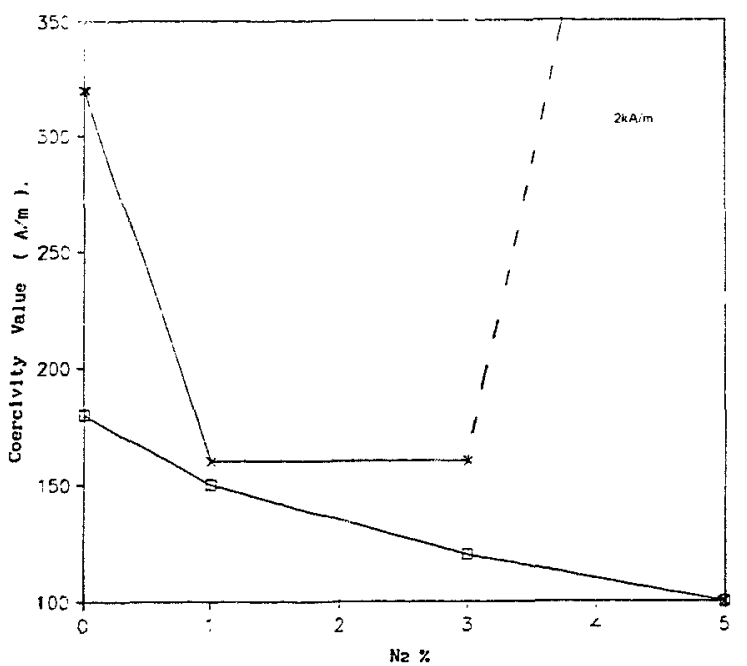

Fig. 4. The dependence of the coercive force value on the nitrogen sputtering gas content. $\square$ : as-sputtered, $\times$ : annealed.

increase figs. 3, 4. Concerning the last parameter, its dependence on the impurity content was expected since it is related to the anisotropic magnetoresistive parameter. However, $\Delta \rho$ is more sensitive to lower impurity contents then $M_{\mathrm{s}}$.

The effect of annealing on the coercive force is strongly related to the initial nitrogen content. $H_{\mathrm{c}}$ is almost unaffected in films with low initial nitrogur content, while it increases in films sputtered in an Ar 5\% $\mathrm{N}_{2}$ gas mixture. As was expected, an increase of this parameter was also observed in NiFe films sputtered in pure argon.

The value of $M_{\mathrm{s}}$ after annealing was slightly low $\Rightarrow r$ in films sputtered in an Ar 5\% $\mathrm{N}_{2}$ gas mixture than in the other films.

\subsection{AES studies}

In this investigation we are interested in the effect of annealing on the depth profile of nitrogen and the $\mathrm{Ni} / \mathrm{Fe}$ ratio. The relative accuracy of nitrogen concentration is much bet:ir when using the $\mathrm{NiFe} / \mathrm{SiN}$ system in which the nitrogen of the silicon nitride is analyzed with a more accurate technique [9]. Using this calibration method we found that increasing the nitrogen sputtering gas content increases the nitrogen film concentration.
Filrs sputtered in an Ar $5 \% \mathrm{~N}_{2}$ gas mixture contain approximately 8 at\% nitrogen.

Fig. 5 shows Auger spectra in a differential form of $\mathrm{NiFe}$ film sputtered in an $\operatorname{Ar} 5 \% \mathrm{~N}_{2}$ gas mixture. This analysis indicates that nitrogen diffuses out during annealing. Carbon and oxygen were detected in the first atomic layers in all films investigated. However, their contents were under the detection limits in the bulk part after 2-3 min sputtering.

More detailed information was obtained from the depth-profile analysis presented in figs. 6 and 7. In nitrogen-doped films, the nitrogen concentration is uniform throughout the film thickness with a slight enrichment at the surface. Similar depth distribution was reported in nitrogen-implanted nickel foils [15]. In annealed films, the
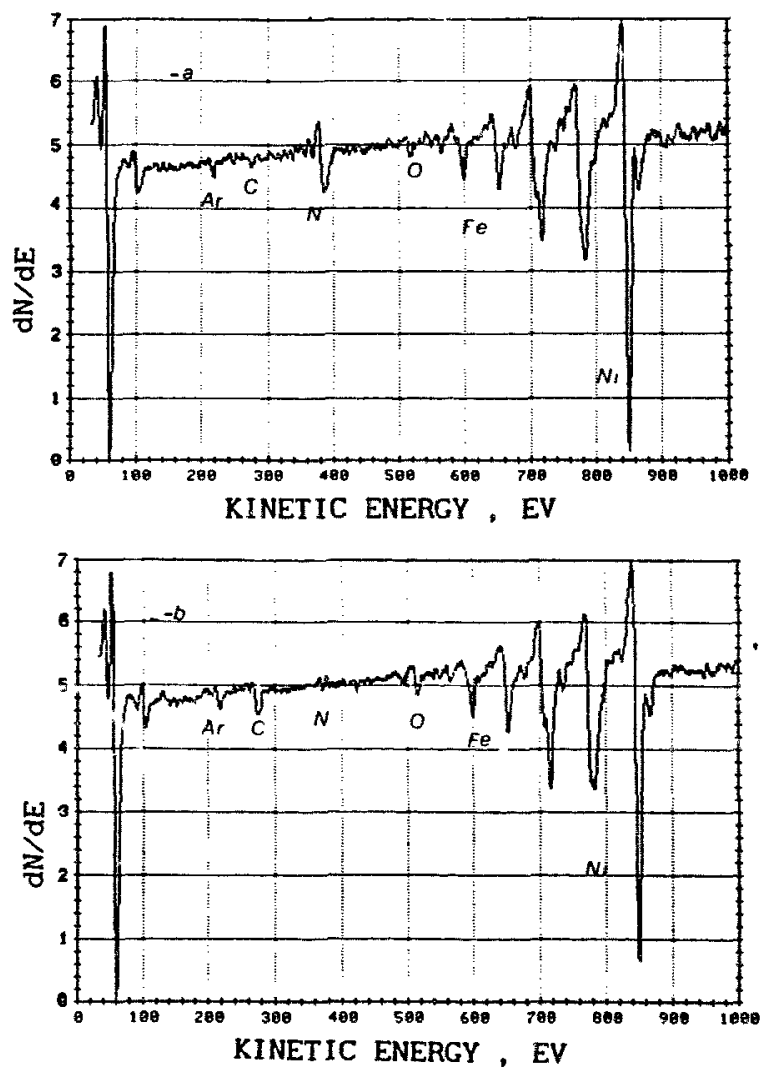

Fig. 5. Auger differential spectrum from a NiFe film sputtered in an Ar $5 \% \mathrm{~N}_{2}$ gas mixture after 2 min surface eroding. (a) As-sputtered, (b) after annealing. 


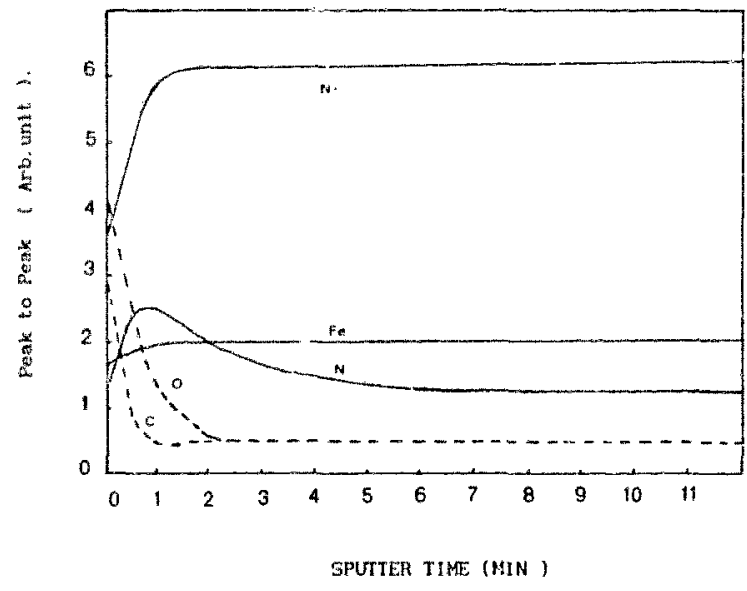

Fig. 6. Depth concentration profiles of as-deposited NiFe films sputtered in an Ar $5 \% \mathrm{~N}_{2}$ gas mixture. Analysis performed with a primary beam of $3 \mathrm{kV}, 0.1 \mu \mathrm{A}$.

nitrogen Auger signal $w: s$ only detectable from the $\mathrm{NiFe}$ /substrate interface and the film's surface indicating that the performed annealing step had promoted nitrogen segregation. From structural and thermodynamic arguments we can expect that segregation occurs at grain boundaries and dislocations.

As reported recently [4], all recorded profiles indicate the presence of three distinct regions: film/substrate interface, bulk and the top surface

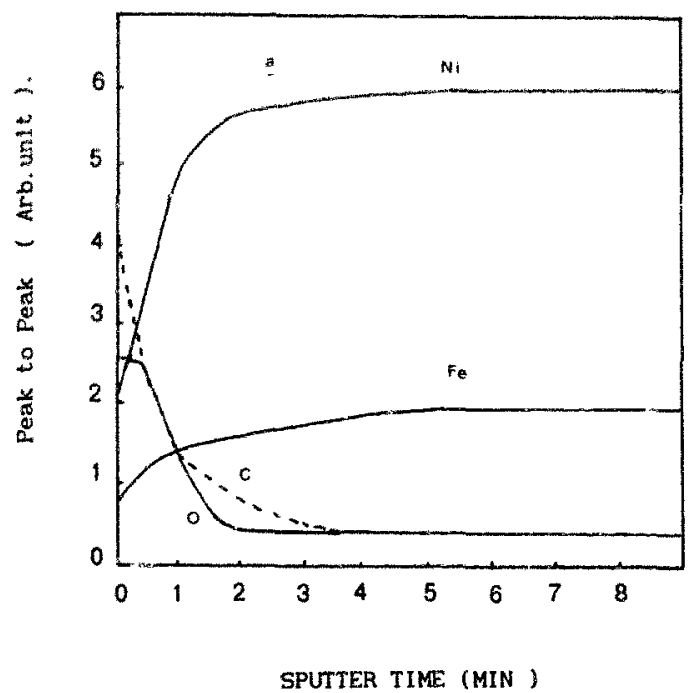

oxide. The top surface of all studied films varies from $2 \mathrm{~nm}$ in as-deposited films to about $4 \mathrm{~nm}$ after annealing. However, the chemical composi tion changes with regard to the presence of nitrogen and the $\mathrm{Ni} / \mathrm{Fe}$ ratio. The lowest ratio corresponding to an incressed iron content, probably in an oxide form, was recorded in an annealed $\mathrm{NiFe}$ film having the highest nitrogen content. The iron segregation at the top layer is in agreement with the observed slight decrease of the saturation magretization. However, it is impossible to detect any iron depletion in the bulk part of the annealed films, using the AES method.

\subsection{TEM observations}

TEM observations were performed in as-deposited and annealed NiFe films, sputtered in $\mathrm{Ar}$ $5 \% \mathrm{~N}_{2}$ plasma. Fig. 8 shows the corresponding TEM bright fieid micrograph and associated diffraction patterns. In as-sputtered film the microstructure consists of very fine grains of $5 \mathrm{~nm}$ diameter having (111) or (200) preferred orientations. In NiFe films sputtered in pure argon the average grain size lies within the range of 10-20 $\mathrm{nm}$ and (111) is the preferred orientation. Besides this, fig. 9 reveals a metastable phase with cubic structure grown locally on a NiFe film. Under a locused microscope beam where the temperature

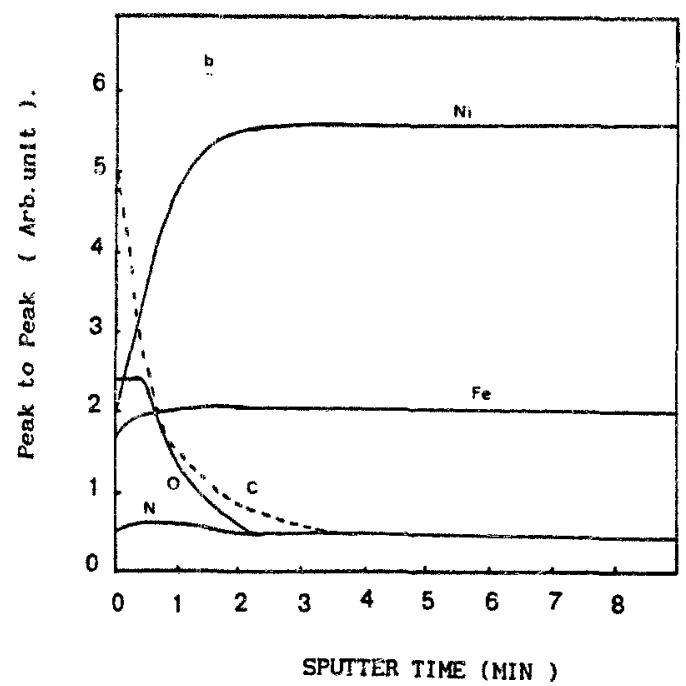

Fig. 7. Depth concentration profiles of annealed NiFe films. (a) Sputtered in pure Ar; (b) sputtered in Ar $5 \% \mathrm{~N}_{2}$ gas mixture. Analys: performed with a primary beam of $3 \mathrm{kV}, 0.1 \mu \mathrm{A}$ 

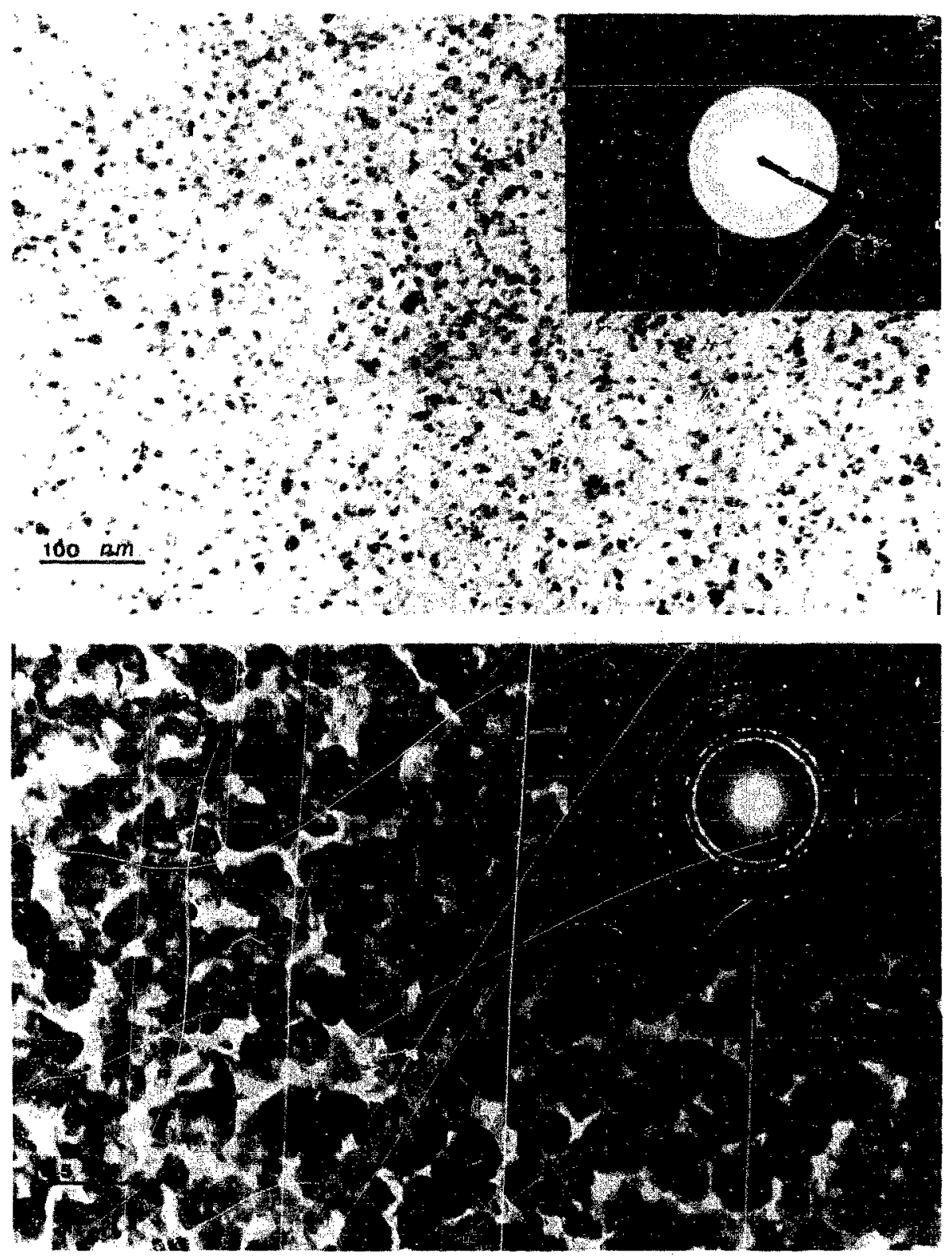

Fig. 8. Transmission electron microscope micrograph and corresponding electron diffraction pattern of as-sputtered (above) and annealed (below) NiFe sputtered in an $\operatorname{Ar} 5 \% \mathrm{~N}_{2}$ ors mixture.

does not exceed $70^{\circ} \mathrm{C}$ this phase was unstable and gradually disappeared with time. We have identified another phase in monocrystalline form, which had a cubic structure and a lattice parameter equal to $0.38 \mathrm{~nm}$ corresponding to the compound
(FeNiN)C [10]. The diffraction images (fig. 10) display the transformation of this phase under focused microscope beam into a polycrystalline structure. The increased surface segregation reyealed with the AES analysis method and the fast 


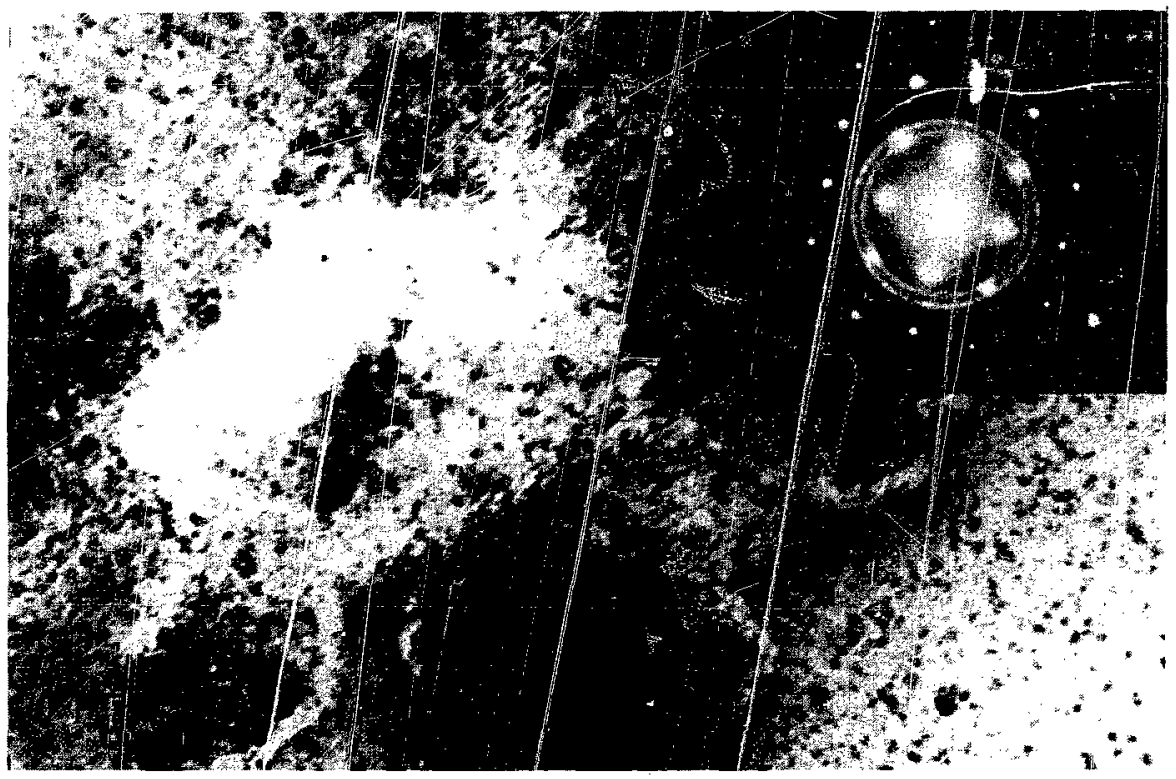

Fig. 9. Transmission electron microscope micrograph and corresponding electron diffraction pattern of NiFe film sputtered in an Ar $5 \% \mathbf{N}_{2}$ gas mixture showing metastable phase.

diffusion of the metalloid elements ( $C, N, O)$, combined with the sputter conditions adopted in our experiments, could be the origin of the growth of these phases. Similar observations were reported recently on the $\mathrm{FeNiCr}$ system [11].

In annealed films the microstructure exhibits different features:

(1) The well known annealed NiFe microstructure is characterized by large grains $(10-20 \mathrm{~nm})$, in which the preferred orientations are still the
(111) and (200). Besides this, new orientations are present suggesting that during annealing grain reorientation has occurred.

(2) Weak rings were detected corresponding to the ordered $\mathrm{Ni}_{3} \mathrm{Fe}$ structure which suggested that an order-disorder transformation had occurred during annealing.

(3) A thin layer with a grain size of $50 \mathrm{~nm}$ covering the annealed microstructure component fig. 8. However, this phase was stable and had a
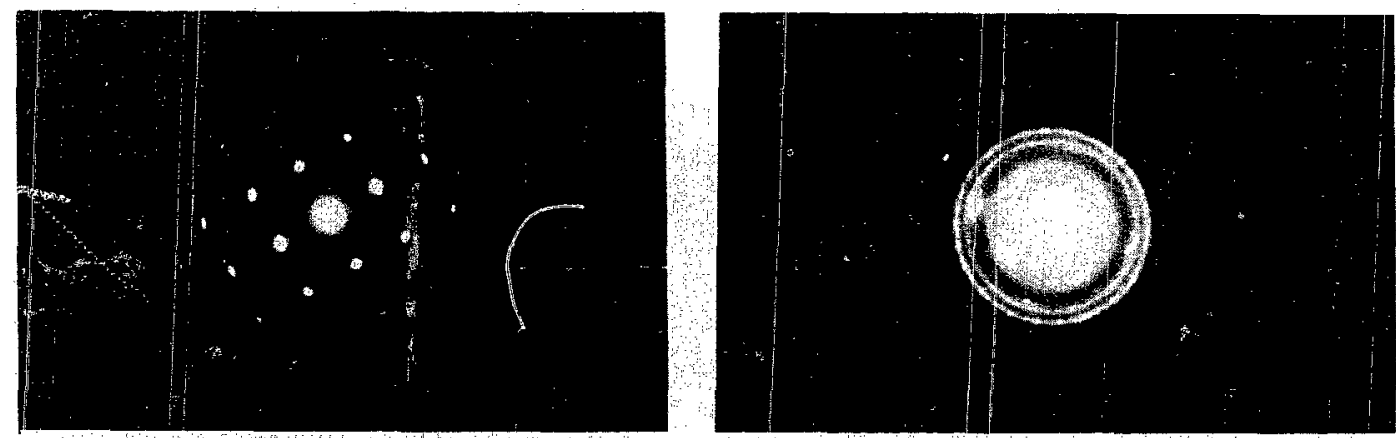

Fig. 10. The electron diffraction patterns of FeNiN (left) as-sputtered, (right) after exposition under a focused microscope beam. 
low symmetry; probably corresponding to an oxide or nitride of $\mathrm{Ni}$ and/or Fe.

\section{Discussion}

Impurities depending on their nature, distribution and interaction energy with the solvent atoms affect thin film microstructure and physical properties differently. In NiFe nitrogen changes the $3 d$-eiectron density at the iron atoms [10]. It is not surprising that it degrades the anisotropic magnetoresistive parameter $\Delta \rho$ and the saturation magnetization $M_{\mathrm{s}}$. The effect of this metalloid element is quite similar to that of many impurities of $3 \mathrm{~d}$ transition metals [6] and it may have consequences for the anisotropy by a change of spin-orbit coupling.

Furthermore the strong sensitivity of $\Delta \rho$ to low nitrogen concentration, in the range where $M_{\mathrm{s}}$ remains unaffected, may explain the conflicting experimental results on $\Delta \rho$ measurements [12], especially in thinner films where the concentration of the metalloid element is high and difficult to control in the deposition systems. Therefore, the dependence of $\Delta \rho$ on the thickness must be investigated under ultrahigh-vacuum conditions.

In annealed films, the surprising increase of $\Delta \rho$ with the initial nitrogen sputtering gas content is not clear. It may be related to the surface transformations during annealing. Nevertheless, the nitrogen segregation to interfaces could also contribute to this.

Both the coercive force and the anisotropy field strength are very important for practical applications. The effect of a low nitrogen addition on the coercivity in as-sputtered films is related to the increase of the preferred orientation (200) and the fine-grain structure. However, in annealed films the role of aitrogen seems to be restricted to decreasing the oxygen content. In fact, segregation of nitrogen to the interface takes place during annealing, as has been reported in AES studies, and probably to the grain boundaries, the exclusive transport mechanism in thin films. Thus any oxidation during annealing is prohibited by the segregated nitrogen leading to low oxygen content and unaffected coercivity. Annealing performed in hydrogen has a similar effect on the magnetic properties of $\mathrm{NiFe}$ which was attributed to a decrease in the film's oxygen content [13]. However, films sputtered with a higher nitrogen gas content exhibit different magnetic properties and microstructures which, in agreement with the experimental results, are related to the iron and nitrogen segregation to the surface. Surface transformations were also reported in annealed $\mathrm{NiCo}$ films sputtered in nitrogen [14], but not attributed to the chemical composition changes.

The accumulation of $\mathrm{Fe}$ in the top layer is the result of nitrogen diffusion via migration of $\mathrm{N}-\mathrm{Fe}$ dumbbells [15]. At the surface the $\mathrm{N}-\mathrm{N}$ bonding also occurs to give molecular nitrogen leaving an iron-nitrogen-rich region, as has been reported in AES studies. Such a mechanism is also operative at the grain boundaries and is probably the origin of the observed ordered structure in annealed films reported in TEM investigations. The high coercivity of these films :s related to surface changes and preferred orientation. Investigation on the diffusion-out kinetics of nitrogen, however, shows that the coercive force decreases with the annealing time until the out-diffusion is complete. This suggests that $H_{c}$ is strongly sensitive to the trapped nitrogen in the grain boundaries and interfaces. $\mathrm{NiFe}$ films sputtered in pure argon and annealed at higher temperatures exhibit similar magnetic properties; in this case segregation is probably induced by grain growth. Such films with a coercive force much higher than the anisotropy field strength are classified as inverted. The origin of their unusual magnetic properties was explained by the anisotropy-center model, which was later considered to be part of the ripple theory [16]. It is attributed to the uniaxial character masked by random perturbing local anisotropies due to inhomogeneities in the film as e.g. randomly orienied crystallites or stresses. These considerations suggest that enhanced segregation or precipitation of compounds as the ordered structure $\mathrm{Ni}_{3} \mathrm{Fe}$ at the grain boundaries introduce additional anisotropy in the matrix. This can be due to exchange or magnetostriction mechanisms.

Further nitrogen addition, like most impurities, results in a more pronounced thickness dependence of the anisotropy field strength $H_{\mathrm{k}}$ [17]. 
Recently, investigation on the origin of the uniaxial anisotropy in evaporated films has shown that it is thicknes dependent and is induced after formation of an isotropic layer called the "dead layer" [18]. In the interpretation of the origin of this layer the impurity factor was neglected. In the present investigation we have shown that annealing weakly magnetic and isotropic nitrogen-doped $\mathrm{NiFe}$ leads to inverted films with a high coercive force and apparent isotropic magnetic properties. These results suggest that the observed dead-layer properties are due to anomalous magnetic properties in very thin films induced by the segregation of impurities at the grain boundaries. The fine grain structure of these films and the higher concentration of impurities near the substrate support the above suggestion. Moreover, an increased coercive force observed in very thin evaporated $\mathrm{NiFe}$ films was recently [19] related to diffusion of impurities during film deposition from the substrate, which is in agreement with our proposition that impurities play an active role in the initial stage of film growth and the resulting physical properties.

\section{Conclusion}

The effect of nitrogen on the microstructure, the magnetic and the electrical properties of 25 $\mathrm{nm}$ thick permalloy was investigated in assputtered films and after annealing at $400^{\circ} \mathrm{C}$ for $15 \mathrm{~min}$. The results obtained were:

(1) In as-sputtered NiFe films, nitrogen affects the structure; the grain size decreases and the amount of (200) preferred orientation increases. The coercive force $H_{c}$, the anisotropy field strength $H_{\mathrm{k}}$, the saturation magnetization $M_{\mathrm{s}}$ and the anisotropic magnetoresistance $\Delta \rho$ decrease.

(2) The physical properties of NiFe films after annealing and out-diffusion of nitrogen were dependent on the initial nitrogen sputtering gas content. $\Delta \rho$ increases remarkably with increasing nitrogen content. The coercive force was unaffected by annealing in low nitrogen content film but increased dramatically with increasing nitrogen concentration. The nitrogen out-diffusion inducing Fe segregation was found to be the operating mechanism for the observed effects.

\section{Acknowledgements}

We would like to thank our colleagues Dr. I. Beyer, Ir. P. de Haan and Dr. L. J. Hanekamp for their advice and technical assistance.

\section{References}

[1] A.K. Jhingan, J. Appl. Phys. 57 (1985) 3991.

[2] K. Krusch, IEEE Trans. Magn. MAG-22 (1986) 626.

[3] A.D. Butherus and S. Nakahara, IEEE Trans. Magn. MAG-21 (1985) 1301.

[4] K. Ounadjela, H. Lefakis, V.S. Speriousu, C. Hwang and P.S. Alexopoulos, J. Appl. Phys. 65 (1989) 1230.

[5] J.A. Leavitt, J. Appl. Phys. 57 (1985) 4195.

[6] T. Miyazaki and T. Ajima, J. Magn. Magn. Mat. 81 (1989) 91.

[7] K. Eijkel, IEEE. Trans. Magn. MAG-24 (1988) 1957.

[8] B. Elm'rabat, J. Beyer and R. Legtenberg, to be published.

[9] S. Bouwstra, R. Legtenberg and Th. J.A. Popma, Techn. Digest Eurosensors 2, Enschede, The Netherlands (1988).

[10] H.J. Goldschmidt and F. Shirane, Phys. Rev. 126 (1962) 49.

[11] S. Malavasi, A. Ouldennaoua, M. Foos and C. Frantz, J. Vac. Sci. Technol. A 5 (1987) 1888.

[12] F.C. Williams and E.N. Mitchel, Jpn. J. Appl. Phys. 7 (1968) 739.

[13] H. Tanabe and M. Kitada, J. Jpn. Inst. Met. 49 (1985) 34.

[14] H. Maeda, J. Appl. Phys. 53 (1986) 3735.

[15] P.D. Ehni and W.N. Unertl, J. Vac. Sci. Technol. A 5 (1987) 1816.

[16] M.S. Cohen, J. Appl. Phys. 33 (1962) 2968.

[17] M. Prutton, Thin Ferromagneiic Film (Butterworths, Washington, 1964) p. 59.

[18] M. Goto, H. Tange and T. Kanimori, J. Magn. Magn. Mat. 62 (1986) 251.

[19] M. Katada and H. Nakamura, Thin Solid Films 167 (1988) L39. 